\title{
CoNLL 2021
}

The 25th Conference on

Computational Natural Language Learning

\section{Proceedings of the Conference}

November $10-11,2021$

Online 


\section{Sponsors}

\section{Google}

(C2021 The Association for Computational Linguistics

Order copies of this and other ACL proceedings from:

Association for Computational Linguistics (ACL)

209 N. Eighth Street

Stroudsburg, PA 18360

USA

Tel: +1-570-476-8006

Fax: +1-570-476-0860

acleaclweb.org

ISBN 978-1-955917-05-6 


\section{Introduction}

Welcome to the 25th Conference on Computational Natural Language Learning (CoNLL). The conference is co-organized with EMNLP this year, but is taking place online, due to the impact of the COVID-19 pandemic.

Following the footsteps of CoNLL 2020, and in departure from previous editions of the conference, CoNLL 2021 focuses on theoretically, cognitively and scientifically motivated approaches to computational linguistics, rather than on work driven by particular engineering applications. This shift was indicated in the call for papers, the instructions given to the program committee, as well as in the publicity about the conference.

We received 226 submissions, of which 202 were sent out for review (the remaining submissions were desk-rejected or withdrawn by the authors). 52 submissions were ultimately accepted for publication (23.0\% acceptance rate). ${ }^{1}$ CoNLL 2021 features two invited talks, by Jennifer Culbertson (The University of Edinburgh, UK) and Gary Lupyan (University of Wisconsin-Madison, USA). We are grateful to all our reviewers (listed on the next page), and our dedicated area chairs: Ryan Cotterell, Adina Williams, Micha Elsner, Allyson Ettinger, Dipendra Misra, Samira Shaikh, Andrew Caines, Roi Reichart, Maja Popović, Rui Wang, Carlos Gómez-Rodríguez, Rob van der Groot, Dieuwke Hupkes, Xin Eric Wang, Michael Roth, Gabriel Stanovsky, Tanmoy Chakraborty, Kyle Gorman

We are also grateful to our publicity chair, Leshem Choshen, and our publication chair, Mareike Hartmann, for their substantial contribution to the conference, and to the SIGNLL information officers, Pieter Fivez and Jens Lemmens.

We would further like to acknowledge the support we received from Google for generously supporting the conference. Finally, we would also like to thank Julia Hockenmaier and Afra Alishahi and the members of the SIGNLL board for entrusting to us the task of organizing the conference, and Raquel Fernández and Tal Linzen, the organizers of CoNLL 2020, for their great advice and support.

Arianna Bisazza and Omri Abend

CoNLL 2021 conference co-chairs

\footnotetext{
${ }^{1}$ When computing the acceptance rate based only on papers sent out for review, the rate becomes $25.7 \%$.
} 



\section{Organizing Committee}

\section{Conference Chairs}

Arianna Bisazza, University of Groningen, The Netherlands

Omri Abend, Hebrew University of Jerusalem, Israel

\section{Invited Speakers}

Jennifer Culbertson, University of Edinburgh, UK

Gary Lupyan, University of Wisconsin-Madison, USA

\section{Area Chairs}

Ryan Cotterell, ETH Zürich, Switzerland

Adina Williams, Facebook AI Research, USA

Micha Elsner, The Ohio State University, USA

Allyson Ettinger, University of Chicago, USA

Dipendra Misra, Microsoft Research, United States

Samira Shaikh, University of North Carolina at Charlotte, USA

Andrew Caines, University of Cambridge, UK

Roi Reichart, Technion - Israel Institute of Technology, Israel

Maja Popović, ADAPT, Dublin City University, Ireland

Rui Wang, Shanghai Jiao Tong University, China

Carlos Gómez-Rodríguez, Universidade da Coruña, Spain

Rob van der Groot, IT University of Copenhagen, Denmark

Dieuwke Hupkes, Facebook AI Research, France

Xin Eric Wang, University of California, Santa Cruz, USA

Michael Roth, University of Stuttgart, Germany

Gabriel Stanovsky, The Hebrew University of Jerusalem, Israel

Tanmoy Chakraborty, Indraprastha Institute of Information Technology Delhi (IIIT-D), India

Kyle Gorman, The Graduate Center, City University of New York, USA

\section{Publication Chair}

Mareike Hartmann, DFKI, Germany

\section{Publicity Chair}

Leshem Choshen, Hebrew University of Jerusalem, Israel

\section{Program Committee}

Ahmed AbuRa'ed, Lasha Abzianidze, Omar Agha, Željko Agić, Alan Akbik, Md. Shad Akhtar, Firoj Alam, Chris Alberti, Afra Alishahi, Mark Anderson, Saba Anwar, Reut Apel, Jun Araki, Christoph Aurnhammer, Eleftherios Avramidis, Fan Bai, Miguel Ballesteros, Yonatan Belinkov, Eyal Ben-David, Ryan Benmalek, Robert Berwick, Shohini Bhattasali, Russa Biswas, Hagen Blix, Valts Blukis, Gosse Bouma, Anouck Braggaar, Jonathan Brennan, Harry Bunt, Davide Buscaldi, Jose Camacho-Collados, Ricardo Campos, Spencer Caplan, Fausto Carcassi, Giovanni Cassani, Sheila Castilho, Rui Chaves, Xinchi Chen, Kehai Chen, Hanjie Chen, Emmanuele Chersoni, Grzegorz Chrupała, Stephen Clark, Jacob Collard, Ailís Cournane, Francisco M Couto, Josep Crego, 
Raj Dabre, Walter Daelemans, Verna Dankers, Amitava Das, Forrest Davis, Aniello De Santo, Mathieu Dehouck, Shrey Desai, Maunendra Sankar Desarkar, Nina Dethlefs, Maria Pia di Buono, Shuoyang Ding, Simon Dobnik, Lucia Donatelli, Rrui Dong, Li Dong, Zi-Yi Dou, Rotem Dror, Andrew Drozdov, Ewan Dunbar, Jonathan Dunn, Yo Ehara, Akiko Eriguchi, Katrin Erk, Luis Espinosa Anke, Amir Feder, Raquel Fernández, Elisa Ferracane, Andrea K. Fischer, George Foster, Abdellah Fourtassi, Stella Frank, Diego Frassinelli, Yoshinari Fujinuma, Richard Futrell, Balaji Ganesan, Marcos Garcia, Phoebe Gaston, Kim Gerdes, Kripabandhu Ghosh, Goran Glavaš, Teresa Gonçalves, Sian Gooding, Kartik Goyal, Alvin Grissom II, Dagmar Gromann, Onur Gungor, Han Guo, Francisco Guzmán, Gholamreza Haffari, Michael Hahn, Sadid A. Hasan, Zhiwei He, Drahomira Herrmannova, Jack Hessel, Bertrand Higy, Cuong Hoang, Cong Duy Vu Hoang, Julia Hockenmaier, Nora Hollenstein, Mark Hopkins, Pedram Hosseini, Jennifer Hu, Tim Hunter, Samar Husain, Alexander Huth, Abhik Jana, Ganesh Jawahar, Lifeng Jin, Jaap Jumelet, Diptesh Kanojia, Alina Karakanta, Casey Kennington, Halil Kilicoglu, Tracy Holloway King, Christo Kirov, Thomas Kober, Parisa Kordjamshidi, Amrith Krishna, Jonathan K. Kummerfeld, Anoop Kunchukuttan, John P. Lalor, Ni Lao, Samuel Läubli, Alberto Lavelli, Phong Le, Jochen L. Leidner, Sarah Ita Levitan, Martha Lewis, Junyi Jessy Li, Tao Li, Constantine Lignos, Tal Linzen, Marina Litvak, Zoey Liu, Nelson F. Liu, Wei Lu, Andreas Maletti, Bruno Martins, David McClosky, R. Thomas McCoy, Kate McCurdy, Stephen McGregor, Parth Mehta, Stephan Meylan, Chenggang Mi, Nicole Mirea, Rahul Mishra, Kanishka Misra, Dipendra Misra, Daichi Mochihashi, Francis Mollica, Manuel Montes, Alessandro Moschitti, Animesh Mukherjee, Philippe Muller, Max Müller-Eberstein, Preslav Nakov, Khanh Nguyen, Massimo Nicosia, Andreas Niekler, Tong Niu, Hiroshi Noji, Brendan O'Connor, Tim O'Gorman, Fredrik Olsson, Yasumasa Onoe, Juri Opitz, Yohei Oseki, Simon Ostermann, Hiroki Ouchi, Jessica Ouyang, Alexandros Papangelis, Chan Young Park, Dan Parker, Alicia Parrish, Debjit Paul, Lisa Pearl, Hao Peng, Laurel Perkins, Sandro Pezzelle, Grusha Prasad, Emily Prud'hommeaux, Ella Rabinovich, Alexandre Rademaker, Daniele P. Radicioni, Taraka Rama, Rohan Ramanath, Alan Ramponi, Ari Rappoport, Shauli Ravfogel, Jonathan Rawski, Sagnik Ray Choudhury, Stefanie Reed, Paolo Rosso, Guy Rotman, Alla Rozovskaya, Maria Ryskina, Mrinmaya Sachan, Kenji Sagae, Hassan Sajjad, Elizabeth Salesky, Vicente Ivan Sanchez Carmona, Germán Sanchis-Trilles, Sashank Santhanam, Giorgio Satta, Asad Sayeed, David Schlangen, Steven Schockaert, William Schuler, Sebastian Schuster, Scott Seyfarth, Samira Shaikh, Cory Shain, Abu Awal Md Shoeb, Miikka Silfverberg, Arabella Sinclair, Mayank Singh, Koustuv Sinha, Kevin Small, Kenny Smith, Richard Sproat, Edward Stabler, Efstathios Stamatatos, Mark Steedman, Karl Stratos, Yoshihiko Suhara, Maartje ter Hoeve, Alberto Testoni, Amalia Todirascu, Gaurav Singh Tomar, Mariya Toneva, Shubham Toshniwal, Reut Tsarfaty, Natalia Tyulina, Ahmet Üstün, Tim Van de Cruys, Oskar van der Wal, Shikhar Vashishth, Prashanth Vijayaraghavan, David Vilar, Alex Warstadt, Taro Watanabe, Noah Weber, Lucas Weber, Colin Wilson, Cai Wingfield, Eduardo Xamena, Xi Ye, Seid Muhie Yimam, François Yvon, Roberto Zamparelli, Marcos Zampieri, Mike Zhang, Zhisong Zhang, Mozhi Zhang, Tianlin Zhang, Mengxuan Zhao, Kai Zhao, Heike Zinsmeister

\section{Outstanding Reviewers}

We would like to recognize the following outstanding reviewers:

Kanishka Mitra, Forrest Davis, Alicia Parrish, Lisa Pearl, Emily Prud'hommeaux, Tom McCoy, Robert Berwick, Mark Anderson, Brendan O'Connor, Hiroshi Noji, Hagen Blix, and Max MüllerEberstein, Lasha Abzianidze, Alan Akbik, Harry Bunt, Emmanuele Chersoni, Katrin Erk, Luis Espinosa Anke, Goran Glavaš, Junyi Jessy Li, Philippe Muller, Andreas Niekler, Tim O'Gorman, Mark Steedman, Tracy Holloway King, Tong Niu, Francisco Guzmán, Sebastian Schuster, Raquel Fernandez, Casey Kennington, Khanh Nguyen, Andrea Fischer, Grzegorz Chrupała, Kate McCurdy, Shauli Ravfogel, Sagnik Ray Choudhury, Verna Dankers, Shuoyang Ding, Guy Rotman, Rotem Dror, Amir Feder, Yoshinari Fujinuma, Ella Rabinovich, Marcos Zampieri 


\section{Table of Contents}

"It's our fault!": Insights Into Users' Understanding and Interaction With an Explanatory Collaborative Dialog System

Katharina Weitz, Lindsey Vanderlyn, Ngoc Thang Vu and Elisabeth André ............... 1

Dependency Induction Through the Lens of Visual Perception

Ruisi Su, Shruti Rijhwani, Hao Zhu, Junxian He, Xinyu Wang, Yonatan Bisk and Graham Neubig 17

VQA-MHUG: A Gaze Dataset to Study Multimodal Neural Attention in Visual Question Answering

Ekta Sood, Fabian Kögel, Florian Strohm, Prajit Dhar and Andreas Bulling ...............27

"It seemed like an annoying woman": On the Perception and Ethical Considerations of Affective Language in Text-Based Conversational Agents

Lindsey Vanderlyn, Gianna Weber, Michael Neumann, Dirk Väth, Sarina Meyer and Ngoc Thang

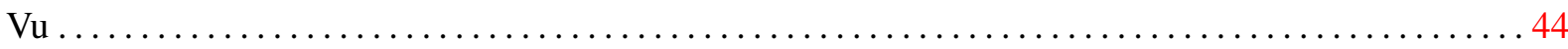

On Language Models for Creoles

Heather Lent, Emanuele Bugliarello, Miryam de Lhoneux, Chen Qiu and Anders Søgaard ..... 58

Do pretrained transformers infer telicity like humans?

Yiyun Zhao, Jian Gang Ngui, Lucy Hall Hartley and Steven Bethard .................... 72

The Low-Dimensional Linear Geometry of Contextualized Word Representations

Evan Hernandez and Jacob Andreas

Generalising to German Plural Noun Classes, from the Perspective of a Recurrent Neural Network

Verna Dankers, Anna Langedijk, Kate McCurdy, Adina Williams and Dieuwke Hupkes........ 94

Can Language Models Encode Perceptual Structure Without Grounding? A Case Study in Color

Mostafa Abdou, Artur Kulmizev, Daniel Hershcovich, Stella Frank, Ellie Pavlick and Anders S $\varnothing-$

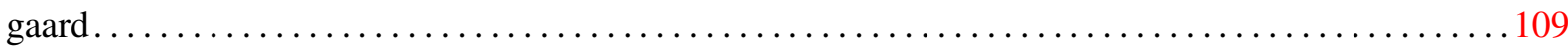

Empathetic Dialog Generation with Fine-Grained Intents

Yubo Xie and Pearl Pu ............................................. 133

Enriching Language Models with Visually-grounded Word Vectors and the Lancaster Sensorimotor Norms

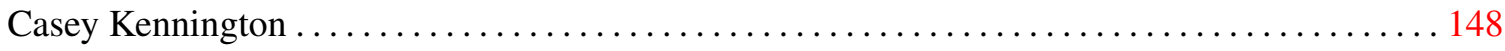

Learning Zero-Shot Multifaceted Visually Grounded Word Embeddings via Multi-Task Training

Hassan Shahmohammadi, Hendrik P. A. Lensch and R. Harald Baayen................. 158

Does language help generalization in vision models?

Benjamin Devillers, Bhavin Choksi, Romain Bielawski and Rufin VanRullen ............. 171

Understanding Guided Image Captioning Performance across Domains

Edwin G. Ng, Bo Pang, Piyush Sharma and Radu Soricut ...

Counterfactual Interventions Reveal the Causal Effect of Relative Clause Representations on Agreement Prediction

Shauli Ravfogel, Grusha Prasad, Tal Linzen and Yoav Goldberg . . 
Who's on First?: Probing the Learning and Representation Capabilities of Language Models on Deterministic Closed Domains

David Demeter and Doug Downey

Data Augmentation of Incorporating Real Error Patterns and Linguistic Knowledge for Grammatical Error Correction

$\mathrm{Xia} \mathrm{Li}$ and Junyi He

Agree to Disagree: Analysis of Inter-Annotator Disagreements in Human Evaluation of Machine Translation Output

Maja Popović

A Multilingual Benchmark for Probing Negation-Awareness with Minimal Pairs

Mareike Hartmann, Miryam de Lhoneux, Daniel Hershcovich, Yova Kementchedjhieva, Lukas

Nielsen, Chen Qiu and Anders Søgaard ......................................... 244

Explainable Natural Language to Bash Translation using Abstract Syntax Tree

Shikhar Bharadwaj and Shirish Shevade ................................. 258

Learned Construction Grammars Converge Across Registers Given Increased Exposure

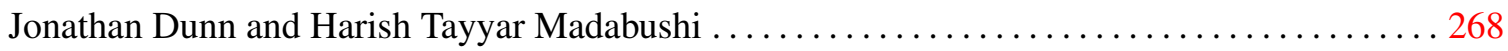

Tokenization Repair in the Presence of Spelling Errors

Hannah Bast, Matthias Hertel and Mostafa M. Mohamed . .

A Coarse-to-Fine Labeling Framework for Joint Word Segmentation, POS Tagging, and Constituent Parsing

Yang Hou, Houquan Zhou, Zhenghua Li, Yu Zhang, Min Zhang, Zhefeng Wang, Baoxing Huai and

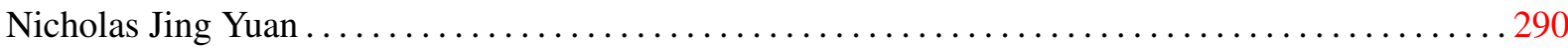

Understanding the Extent to which Content Quality Metrics Measure the Information Quality of Summaries

Daniel Deutsch and Dan Roth.

Summary-Source Proposition-level Alignment: Task, Datasets and Supervised Baseline

Ori Ernst, Ori Shapira, Ramakanth Pasunuru, Michael Lepioshkin, Jacob Goldberger, Mohit Bansal

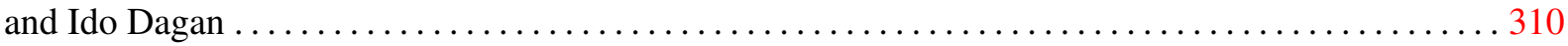

Exploring Metaphoric Paraphrase Generation

Kevin Stowe, Nils Beck and Iryna Gurevych

Imposing Relation Structure in Language-Model Embeddings Using Contrastive Learning

Christos Theodoropoulos, James Henderson, Andrei Catalin Coman and Marie-Francine Moens337

NOPE: A Corpus of Naturally-Occurring Presuppositions in English

Alicia Parrish, Sebastian Schuster, Alex Warstadt, Omar Agha, Soo-Hwan Lee, Zhuoye Zhao,

Samuel R. Bowman and Tal Linzen .......................................... 349

Pragmatic competence of pre-trained language models through the lens of discourse connectives

Lalchand Pandia, Yan Cong and Allyson Ettinger ................................... 367

Predicting Text Readability from Scrolling Interactions

Sian Gooding, Yevgeni Berzak, Tony Mak and Matt Sharifi 380 
Modeling the Interaction Between Perception-Based and Production-Based Learning in Children's Early Acquisition of Semantic Knowledge

Mitja Nikolaus and Abdellah Fourtassi

Scaffolded input promotes atomic organization in the recurrent neural network language model

Philip A. Huebner and Jon A. Willits . . . . . . . . . . . . . . . . . . . . . . . . 408

Grammatical Profiling for Semantic Change Detection

Andrey Kutuzov, Lidia Pivovarova and Mario Giulianelli

Deconstructing syntactic generalizations with minimalist grammars

Marina Ermolaeva .............................................. 435

Relation-aware Bidirectional Path Reasoning for Commonsense Question Answering

Junxing Wang, Xinyi Li, Zhen Tan, Xiang Zhao and Weidong Xiao ...

Does referent predictability affect the choice of referential form? A computational approach using masked coreference resolution

Laura Aina, Xixian Liao, Gemma Boleda and Matthijs Westera . 454

Polar Embedding

Ran Iwamoto, Ryosuke Kohita and Akifumi Wachi

Commonsense Knowledge in Word Associations and ConceptNet

Chunhua Liu, Trevor Cohn and Lea Frermann

Cross-document Event Identity via Dense Annotation

Adithya Pratapa, Zhengzhong Liu, Kimihiro Hasegawa, Linwei Li, Yukari Yamakawa, Shikun

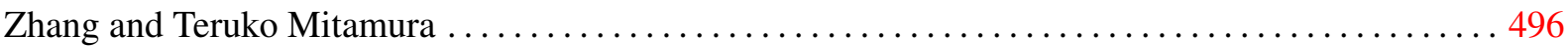

Tackling Zero Pronoun Resolution and Non-Zero Coreference Resolution Jointly

Shisong Chen, Binbin Gu, Jianfeng Qu, Zhixu Li, An Liu, Lei Zhao and Zhigang Chen ...... 518

Negation-Instance Based Evaluation of End-to-End Negation Resolution

Elizaveta Sineva, Stefan Grünewald, Annemarie Friedrich and Jonas Kuhn

Controlling Prosody in End-to-End TTS: A Case Study on Contrastive Focus Generation

Siddique Latif, Inyoung Kim, Ioan Calapodescu and Laurent Besacier .................. 544

A Large-scale Comprehensive Abusiveness Detection Dataset with Multifaceted Labels from Reddit

Hoyun Song, Soo Hyun Ryu, Huije Lee and Jong Park .......................... 552

MirrorWiC: On Eliciting Word-in-Context Representations from Pretrained Language Models

Qianchu Liu, Fangyu Liu, Nigel Collier, Anna Korhonen and Ivan Vulić ................ 562

A Data Bootstrapping Recipe for Low-Resource Multilingual Relation Classification

Arijit Nag, Bidisha Samanta, Animesh Mukherjee, Niloy Ganguly and Soumen Chakrabarti...575

FAST: A carefully sampled and cognitively motivated dataset for distributional semantic evaluation

Stefan Evert and Gabriella Lapesa ....................................... 588

Automatic Error Type Annotation for Arabic

Riadh Belkebir and Nizar Habash ........................................ 596

The Emergence of the Shape Bias Results from Communicative Efficiency

Eva Portelance, Michael C. Frank, Dan Jurafsky, Alessandro Sordoni and Romain Laroche . . 607 
BabyBERTa: Learning More Grammar With Small-Scale Child-Directed Language

Philip A. Huebner, Elior Sulem, Fisher Cynthia and Dan Roth. .

Analysing Human Strategies of Information Transmission as a Function of Discourse Context

Mario Giulianelli and Raquel Fernández . . .

Predicting non-native speech perception using the Perceptual Assimilation Model and state-of-the-art acoustic models

Juliette Millet, Ioana Chitoran and Ewan Dunbar. 661

The Influence of Regional Pronunciation Variation on Children's Spelling and the Potential Benefits of Accent Adapted Spellcheckers

Emma O’Neill, Joe Kenny, Anthony Ventresque and Julie Carson-Berndsen 674 


\section{Conference Program}

Wednesday, November 10, 2021

10:00-10:10 Welcome

10:10-11:30 Oral session 1: Interaction, dialogue, and grounded language learning

10:10-10:30 "It's our fault!": Insights Into Users' Understanding and Interaction With an Explanatory Collaborative Dialog System

Katharina Weitz, Lindsey Vanderlyn, Ngoc Thang Vu and Elisabeth André

10:30-10:50 Dependency Induction Through the Lens of Visual Perception

Ruisi Su, Shruti Rijhwani, Hao Zhu, Junxian He, Xinyu Wang, Yonatan Bisk and Graham Neubig

10:50-11:10 VQA-MHUG: A Gaze Dataset to Study Multimodal Neural Attention in Visual Question Answering

Ekta Sood, Fabian Kögel, Florian Strohm, Prajit Dhar and Andreas Bulling

11:10-11:30 "It seemed like an annoying woman": On the Perception and Ethical Considerations of Affective Language in Text-Based Conversational Agents

Lindsey Vanderlyn, Gianna Weber, Michael Neumann, Dirk Väth, Sarina Meyer and Ngoc Thang Vu

\section{1:30-12:00 Break}

12:00-13:10 Keynote I: Linking learning to language typology

Jennifer Culbertson 


\section{Wednesday, November 10, 2021 (continued)}

\section{3:10-14:10 Lunch break}

14:10-15:50 Oral session 2: Theoretical analysis, probing, and interpretation of language models

14:10-14:30 On Language Models for Creoles

Heather Lent, Emanuele Bugliarello, Miryam de Lhoneux, Chen Qiu and Anders Søgaard

14:30-14:50 Do pretrained transformers infer telicity like humans?

Yiyun Zhao, Jian Gang Ngui, Lucy Hall Hartley and Steven Bethard

14:50-15:10 The Low-Dimensional Linear Geometry of Contextualized Word Representations Evan Hernandez and Jacob Andreas

15:10-15:30 Generalising to German Plural Noun Classes, from the Perspective of a Recurrent Neural Network

Verna Dankers, Anna Langedijk, Kate McCurdy, Adina Williams and Dieuwke Hupkes

15:30-15:50 Can Language Models Encode Perceptual Structure Without Grounding? A Case Study in Color

Mostafa Abdou, Artur Kulmizev, Daniel Hershcovich, Stella Frank, Ellie Pavlick and Anders Søgaard

\section{5:50-16:20 Break}

\section{6:20-18:00 Poster session 1}

16:20-18:00 Empathetic Dialog Generation with Fine-Grained Intents

Yubo Xie and Pearl Pu

16:20-18:00 Enriching Language Models with Visually-grounded Word Vectors and the Lancaster Sensorimotor Norms

Casey Kennington

16:20-18:00 Learning Zero-Shot Multifaceted Visually Grounded Word Embeddings via MultiTask Training

Hassan Shahmohammadi, Hendrik P. A. Lensch and R. Harald Baayen 


\section{Wednesday, November 10, 2021 (continued)}

16:20-18:00 Does language help generalization in vision models?

Benjamin Devillers, Bhavin Choksi, Romain Bielawski and Rufin VanRullen

16:20-18:00 Understanding Guided Image Captioning Performance across Domains

Edwin G. Ng, Bo Pang, Piyush Sharma and Radu Soricut

16:20-18:00 Counterfactual Interventions Reveal the Causal Effect of Relative Clause Representations on Agreement Prediction

Shauli Ravfogel, Grusha Prasad, Tal Linzen and Yoav Goldberg

16:20-18:00 Who's on First?: Probing the Learning and Representation Capabilities of Language Models on Deterministic Closed Domains

David Demeter and Doug Downey

16:20-18:00 Data Augmentation of Incorporating Real Error Patterns and Linguistic Knowledge for Grammatical Error Correction

Xia Li and Junyi He

16:20-18:00 Agree to Disagree: Analysis of Inter-Annotator Disagreements in Human Evaluation of Machine Translation Output

Maja Popović

16:20-18:00 A Multilingual Benchmark for Probing Negation-Awareness with Minimal Pairs Mareike Hartmann, Miryam de Lhoneux, Daniel Hershcovich, Yova Kementchedjhieva, Lukas Nielsen, Chen Qiu and Anders Søgaard

16:20-18:00 Explainable Natural Language to Bash Translation using Abstract Syntax Tree Shikhar Bharadwaj and Shirish Shevade

16:20-18:00 Learned Construction Grammars Converge Across Registers Given Increased Exposure

Jonathan Dunn and Harish Tayyar Madabushi

16:20-18:00 Tokenization Repair in the Presence of Spelling Errors

Hannah Bast, Matthias Hertel and Mostafa M. Mohamed

16:20-18:00 A Coarse-to-Fine Labeling Framework for Joint Word Segmentation, POS Tagging, and Constituent Parsing

Yang Hou, Houquan Zhou, Zhenghua Li, Yu Zhang, Min Zhang, Zhefeng Wang, Baoxing Huai and Nicholas Jing Yuan

16:20-18:00 Understanding the Extent to which Content Quality Metrics Measure the Information Quality of Summaries

Daniel Deutsch and Dan Roth 
Thursday, November 11, 2021

10:00-11:40 Oral session 3: Lexical, compositional, and discourse semantics; Pragmatics

10:00-10:20 Summary-Source Proposition-level Alignment: Task, Datasets and Supervised Baseline

Ori Ernst, Ori Shapira, Ramakanth Pasunuru, Michael Lepioshkin, Jacob Goldberger, Mohit Bansal and Ido Dagan

10:20-10:40 Exploring Metaphoric Paraphrase Generation

Kevin Stowe, Nils Beck and Iryna Gurevych

10:40-11:00 Imposing Relation Structure in Language-Model Embeddings Using Contrastive Learning

Christos Theodoropoulos, James Henderson, Andrei Catalin Coman and MarieFrancine Moens

11:00-11:20 NOPE: A Corpus of Naturally-Occurring Presuppositions in English

Alicia Parrish, Sebastian Schuster, Alex Warstadt, Omar Agha, Soo-Hwan Lee, Zhuoye Zhao, Samuel R. Bowman and Tal Linzen

11:20-11:40 Pragmatic competence of pre-trained language models through the lens of discourse connectives

Lalchand Pandia, Yan Cong and Allyson Ettinger

\section{1:40-13:20 Poster session 2}

11:40-13:20 Predicting Text Readability from Scrolling Interactions

Sian Gooding, Yevgeni Berzak, Tony Mak and Matt Sharifi

11:40-13:20 Modeling the Interaction Between Perception-Based and Production-Based Learning in Children's Early Acquisition of Semantic Knowledge

Mitja Nikolaus and Abdellah Fourtassi

11:40-13:20 Scaffolded input promotes atomic organization in the recurrent neural network language model

Philip A. Huebner and Jon A. Willits

11:40-13:20 Grammatical Profiling for Semantic Change Detection

Andrey Kutuzov, Lidia Pivovarova and Mario Giulianelli

11:40-13:20 Deconstructing syntactic generalizations with minimalist grammars

Marina Ermolaeva 


\section{Thursday, November 11, 2021 (continued)}

11:40-13:20 Relation-aware Bidirectional Path Reasoning for Commonsense Question Answering

Junxing Wang, Xinyi Li, Zhen Tan, Xiang Zhao and Weidong Xiao

11:40-13:20 Does referent predictability affect the choice of referential form? A computational approach using masked coreference resolution

Laura Aina, Xixian Liao, Gemma Boleda and Matthijs Westera

11:40-13:20 Polar Embedding

Ran Iwamoto, Ryosuke Kohita and Akifumi Wachi

11:40-13:20 Commonsense Knowledge in Word Associations and ConceptNet

Chunhua Liu, Trevor Cohn and Lea Frermann

11:40-13:20 Cross-document Event Identity via Dense Annotation

Adithya Pratapa, Zhengzhong Liu, Kimihiro Hasegawa, Linwei Li, Yukari Yamakawa, Shikun Zhang and Teruko Mitamura

11:40-13:20 Tackling Zero Pronoun Resolution and Non-Zero Coreference Resolution Jointly Shisong Chen, Binbin Gu, Jianfeng Qu, Zhixu Li, An Liu, Lei Zhao and Zhigang Chen

11:40-13:20 Negation-Instance Based Evaluation of End-to-End Negation Resolution Elizaveta Sineva, Stefan Grünewald, Annemarie Friedrich and Jonas Kuhn

11:40-13:20 Controlling Prosody in End-to-End TTS: A Case Study on Contrastive Focus Generation

Siddique Latif, Inyoung Kim, Ioan Calapodescu and Laurent Besacier

11:40-13:20 A Large-scale Comprehensive Abusiveness Detection Dataset with Multifaceted Labels from Reddit

Hoyun Song, Soo Hyun Ryu, Huije Lee and Jong Park

11:40-13:20 MirrorWiC: On Eliciting Word-in-Context Representations from Pretrained Language Models

Qianchu Liu, Fangyu Liu, Nigel Collier, Anna Korhonen and Ivan Vulić

11:40-13:20 A Data Bootstrapping Recipe for Low-Resource Multilingual Relation Classification

Arijit Nag, Bidisha Samanta, Animesh Mukherjee, Niloy Ganguly and Soumen Chakrabarti

11:40-13:20 FAST: A carefully sampled and cognitively motivated dataset for distributional semantic evaluation

Stefan Evert and Gabriella Lapesa 
Thursday, November 11, 2021 (continued)

11:40-13:20 Automatic Error Type Annotation for Arabic

Riadh Belkebir and Nizar Habash

\section{3:20-14:20 Lunch break}

14:20-15:30 Keynote II: What are we learning from language?

Gary Lupyan

\section{5:30-15:50 Break}

15:50-16:50 Oral session 4: Language evolution, acquisition and linguistic theories

15:50-16:10 The Emergence of the Shape Bias Results from Communicative Efficiency

Eva Portelance, Michael C. Frank, Dan Jurafsky, Alessandro Sordoni and Romain Laroche

16:10-16:30 BabyBERTa: Learning More Grammar With Small-Scale Child-Directed Language Philip A. Huebner, Elior Sulem, Fisher Cynthia and Dan Roth

16:30-16:50 Analysing Human Strategies of Information Transmission as a Function of Discourse Context

Mario Giulianelli and Raquel Fernández

16:50-17:10 Break 
Thursday, November 11, 2021 (continued)

\section{7:10-17:50 Oral session 5: Speech and phonology}

17:10-17:30 Predicting non-native speech perception using the Perceptual Assimilation Model and state-of-the-art acoustic models

Juliette Millet, Ioana Chitoran and Ewan Dunbar

17:30-17:50 The Influence of Regional Pronunciation Variation on Children's Spelling and the Potential Benefits of Accent Adapted Spellcheckers

Emma O’Neill, Joe Kenny, Anthony Ventresque and Julie Carson-Berndsen

\section{7:50-18:20 Best Paper Award and Closing Words}




\title{
Keynote Talk I
}

\section{Linking Learning to Language Typology}

\author{
Jennifer Culbertson, The University of Edinburgh, UK
}

One of the most controversial hypotheses in linguistics is that individual-level biases in learning shape language typology at the population-level. While this hypothesis has been around a long time, it has often been supported by less than robust empirical evidence. In this talk, I present a number of studies aimed at providing new sources of evidence linking learning to key features of language. In the first part of the talk, I focus on a classic set of "language universals" which describe common word order patterns. One such pattern is word order harmony, the tendency for syntactic heads and dependents to align across phrases within a language. While harmony has long been claimed to have some special cognitive status, there is also compelling evidence that it may be driven by cognition-external processes of language change. I show that harmony is in fact favoured during learning, influencing how adults and children make inferences under noisy learning conditions, and how they extrapolate to new constructions. I then turn to a more complex pattern of word order which has been proposed to derive from constraints on syntactic representations. I report experimental and quantitative corpus-based evidence to suggest an alternative explanation of this pattern, but one nevertheless driven by learning. In the second part of the talk, I discuss the role of learning in shaping morphosynactic patterns like grammatical gender. I argue that the different biases of children and adults during learning work together to constrain how such patterns emerge and change over time. Finally, I discuss the implications of this work for linguistic theories and models of language learning. 
Keynote Talk II

\title{
What are we Learning from Language?
}

\author{
Gary Lupyan, University of Wisconsin-Madison, USA
}

Where does semantic knowledge come from? Previous work on semantic knowledge within cognitive science has focused on studying knowledge acquired from direct experience with the world and through inference. But recent advances in natural language processing combined with greater availability of large text corpora have revealed that languages encode far more semantic information than previously suspected. In some cases, knowledge that was thought to require direct perceptual experience or inferential reasoning can be derived entirely from language itself. I will present some recent investigations of this idea showing, for example, that embedded within the distributional structure of language is substantial information about visual appearance that people can rely on to learn about what things look like. I will also discuss how distributional semantics are informing our understanding of cross-linguistic differences in word meanings, and the relationship between language and thought. I will end by speculating that the robust availability of linguistic information may conceal radical diversity in human cognition. 